\title{
Evaluation of serum ferritin and some metal elements in type 2 diabetes mellitus patients: comparative cross-sectional study
}

This article was published in the following Dove Press journal:

Diabetes, Metabolic Syndrome and Obesity:Targets and Therapy

2 December 2016

Number of times this article has been viewed

\author{
Amare Desalegn Wolide' \\ Belay Zawdie ${ }^{2}$ \\ Tilahun Alemayehu ${ }^{3}$ \\ Samuel Tadesse' \\ 'Department of Medical Physiology, \\ 2Department of Medical Biochemistry, \\ ${ }^{3}$ Department of Human Anatomy, \\ College of Health Sciences, Jimma \\ University, Jimma, Ethiopia
}

Background: The chronic hyperglycemia of diabetes has been associated with an imbalance of some trace metal elements in the blood sample of type 2 diabetes patients.

Aim: To evaluate the status of serum ferritin and some selected metal elements among type 2 diabetes mellitus (T2DM) patients.

Methods: Facility-based comparative cross-sectional study was conducted from February 15, 2015 to October 30, 2015, at Jimma University Specialized Hospital, Ethiopia. A total of 428 type 2 diabetes and nondiabetes study subjects were recruited to the study. After overnight fasting, $10 \mathrm{~mL}$ of venous blood samples were taken for biochemical and trace metal element analysis. Data were entered into EpiData version 3.5.1 and exported to SPSS version 20 for Windows for analysis.

Results: Serum concentration of $\mathrm{Zn}^{+2}, \mathrm{Mg}^{+2}, \mathrm{Cr}^{+3}$, ferritin, and $\mathrm{Fe}^{+3}$ in patients with type 2 diabetes was significantly lower $(p<0.0001)$ than nondiabetes patients. In contrast, serum $\mathrm{Cu}^{+2}$ was significantly higher $(p<0.0001)$ in type 2 diabetes patients than nondiabetics. In addition, significant differences were not seen in both groups with regard to serum $\mathrm{Mn}^{+2}, \mathrm{Ca}^{+2}$, and $\mathrm{Po}_{4}^{-3}$. Waist-to-hip ratio (WHR), serum $\mathrm{Fe}^{+3}$, ferritin, and $\mathrm{Mn}^{+2}$ were significantly higher among oral hypoglycemic agent users of type 2 diabetes patients than the injectable insulin users. Serum $\mathrm{Zn}^{+2}$ had significant positive correlation with serum $\mathrm{Mg}^{+2}(r=0.738), \mathrm{Cr}^{+3}(r=0.233), \mathrm{Ca}^{+2}(r=0.238)$, and $\mathrm{Po}_{4}^{-3}(r=0.222)$. In addition, serum $\mathrm{Zn}^{+2}$ had shown significant and negative correlation with body mass index (BMI, $r=-0.331$ ), WHR ( $r=-0.340$ ), and fasting blood glucose (FBG, $r=-0.186$ ). Likewise, serum $\mathrm{Mg}^{+2}$ and $\mathrm{Po}_{4}^{-3}$ are significantly and negatively correlated with BMI, WHR, and FBG.

Conclusion: The imbalance of trace metal elements in the blood sample of diabetes is uncertain. Thus, we recommend a prospective cohort study to find out the principal factors behind the problem.

Keywords: diabetes, ferritin, trace metal elements, hyperglycemia, insulin, metal

\section{Background}

Trace metal elements are naturally occurring inorganic elements and have been identified as potential candidates for improving metabolic disorders, because they are involved in prosthetic groups of many proteins and cofactors of many enzymes. ${ }^{1}$ In the last decades, many research studies have reported frequently the direct correlation of diabetes and an imbalance of some trace metal elements. ${ }^{2,3}$ The chronic hyperglycemia of diabetes is associated with long-term damage, dysfunction, and failure of different organs, especially the eyes, kidneys, nerves, heart, and blood vessels. ${ }^{4}$ Mortality attributable to diabetes accounts for $2 \%-3 \%$ of deaths in poor countries and by $2030, \sim 8 \%$ of the adult population is estimated to have diabetes. ${ }^{5}$ According to the International Diabetes Federation, there were over 1.33 million

Diabetes, Metabolic Syndrome and Obesity:Targets and Therapy 2016:9 4I 7-424

417

(c) (1) (\$) 2016 Wolide et al. This work is published and licensed by Dove Medical Press Limited. The full terms of this license are available at https://www.dovepress.com/terms. BY NC php and incorporate the Creative Commons Attribution - Non Commercial (unported, v3.0) License (http://creativecommons.org/licenses/by-nc/3.0/). By accessing the work you hereby accept the Terms. Non-commercial uses of the work are permitted without any further permission from Dove Medical Press Limited, provided the work is properly attributed. For permission for commercial use of this work, please see paragraphs 4.2 and 5 of our Terms (https://www.dovepress.com/terms.php). 
cases of diabetes in Ethiopia during 2015, and the number of deaths due to diabetes was $23,145 .{ }^{6}$ Owing to the increasing prevalence of diabetes, multidisciplinary study aimed at preventing and treating diabetes is one of the worldwide research priorities. ${ }^{7}$ The burden of diabetes and other chronic noncommunicable diseases in Ethiopia has been grossly overlooked by concerned bodies as a consequence of government's intense focus on communicable disease and malnutrition agendas. The few studies done on diabetes were confined on the issue of quality of care and patients satisfaction in the follow-up clinics. Thus, research on diabetes in this country can only be described as inadequate. To remedy this situation, research on the causation aspect of the disease needs to be conducted. To the best of our knowledge, the relationship between diabetic hyperglycemia and relevant blood chemistry and trace metal elements has not yet been investigated in Ethiopia. The study results may contribute to the development of evidence-based decision and intervention strategies to enhance a healthier lifestyles and better diabetes care. Therefore, this study is aimed to evaluate the status of serum ferritin and some selected trace metal elements, such as zinc $\left(\mathrm{Zn}^{+2}\right)$, magnesium $\left(\mathrm{Mg}^{+2}\right)$, chromium $\left(\mathrm{Cr}^{+3}\right)$, iron $\left(\mathrm{Fe}^{+3}\right)$, copper $\left(\mathrm{Cu}^{+2}\right)$, manganese $\left(\mathrm{Mn}^{+2}\right)$, calcium $\left(\mathrm{Ca}^{+2}\right)$, and phosphorus $\left(\mathrm{Po}_{4}^{-3}\right)$, among type 2 diabetes mellitus (T2DM) patients at Jimma University Specialized Hospital (JUSH), Ethiopia.

\section{Subjects and method Study subjects and design}

Comparative cross-sectional study was used among 214 patients with T2DM and 214 age, sex, and educational level matched nondiabetes individuals who were in the outpatient department at JUSH using consecutive sampling technique. The study was conducted in JUSH from February 15, 2015 to October 30, 2015. JUSH is one of the oldest public hospitals in the country, located about $350 \mathrm{~km}$ southwest of the capital, Addis Ababa.

\section{Inclusion and exclusion criteria}

All patients aged 20-79 years with a history of diabetes more than 3 years from their diagnosis at the time of data collection were included. Subjects with a history of 1) pregnancy and lactation, 2) use of any drugs affecting electrolytes at the time of data collection, 3 ) taking nutritional supplements, such as magnesium-containing laxatives, 4) chronic disorders of the liver, kidney, and cardiovascular system, 5) endocrine disorders, 6) established psychiatric disorder and on antidepressant and/or antipsychotic therapy, 7) HIV/AIDS, 8) malignancy, and 9) substance abuse were excluded from the study. Study subjects in the nondiabetes group were included and excluded based on the same criteria as stated earlier.

\section{Ethical consideration}

Prior to participation for the study, written informed consent was obtained from the study subjects. The purpose of the study was clearly described to the study subjects including the benefits and risks of the study. Information concerning the study subjects was kept confidential. The specimen collected from the study subjects was only analyzed for the intended purposes. The study was performed in accordance with the ethical principles outlined in the Helsinki Declaration ${ }^{8}$ and guidelines for Good Clinical Practice. ${ }^{9}$ Ethical approval was obtained from the Jimma University ethical review board. Letter for permission to conduct the study was obtained from JUSH medical director office.

\section{Data collection}

Each study subjects was interviewed and completed the questionnaire in the presence and assistance of trained data collectors (Medical Doctors and Laboratory Technologist). Information concerning age, sex, family history of diabetes, history of hypertension and dyslipidemia, and other information was collected using questionnaire. Anthropometric measures included height and weight; waist and hip circumference; respiratory rate; examination of extremities, eye, skin, and sensory-motor functionality and all routine investigations were measured according to standard protocols. Blood pressure and heart rate were measured three times every 2 minutes after an initial 10-minute rest period.

\section{Specimen collection and storage}

Overnight fasting venous blood $(10 \mathrm{~mL})$ was taken from the median cubital vein of subjects using sterile disposable plastic syringe and preserved in ethylenediamine tetraacetic acid (EDTA. $\mathrm{K}_{3}$ )-containing vacuum tubes (Weihai Hongye, Weihai, People's Republic of China) and plain vacutainer tubes (Shandong Branden Medical Devices Co., Ltd, Beijing, People's Republic of China). Samples in the EDTA. $\mathrm{K}_{3}$-containing vacuum tubes were sent to JUSH Laboratory Department for fasting blood glucose (FBG) determination. Samples in the plain vacutainer tubes were immediately brought to the Medical Physiology Laboratory of Jimma University for processing. These samples were centrifuged at $3000 \mathrm{rpm}$ for 10 minutes, and the serum $(3.2 \mathrm{~mL})$ was transferred to labeled $3.6 \mathrm{~mL} \mathrm{Nunc}^{\mathrm{TM}}$ cryogenic tubes (NUNC INC) and stored at $-20^{\circ} \mathrm{C}$ until analysis. 


\section{Specimen preparation and analysis}

Fasting plasma glucose was determined by glucose hexokinase method (ABX Pentra Glucose hexokinase 13CP kit). Serum C-reactive protein (CRP; ABX Pentra CRP 12CP kit) and ferritin ( $\mathrm{ABX}$ Pentra ferritin 5CP kit) were determined by radioimmunoassay method. Both clinical methods were performed using automated chemistry analyzer ABX Pentra 400 (HORIBA Medical Diagnostics Instruments \& Systems, Montpellier, France). Trace element levels were determined using wet acid digestion method. ${ }^{10-12}$ All sample containers were meticulously washed with nonionic detergents solution and rinsed triply with distilled water and dried in the oven at $80^{\circ} \mathrm{C}$ for 24 hours. Each sample was mixed with $3 \mathrm{~mL}$ concentrated nitric acid and hydrogen peroxide $\left[\mathrm{HNO}_{3}-\mathrm{H}_{2} \mathrm{O}_{2}\right]$ solution $(2: 1, \mathrm{v} / \mathrm{v})$ in a beaker for 10 minutes and heated at $120^{\circ} \mathrm{C}$ on a hot plate until complete digestion had taken place. The beaker was loosely covered by $150 \mathrm{~mm}$ Pyrex watch glass to avoid contamination from the air. Then, the samples were brought to $5.4 \mathrm{~mL}$ and allowed to cool at room temperature while continuously adding triple distilled water to maintain the volume. After cooling down, the digested samples were centrifuged at 10,000 rpm for 30 seconds and the organic supernatant aspirated directly into the flam. Finally, the clear samples were analyzed for trace elements using atomic absorption spectrophotometry (Shimadzu AA-670, Kyoto, Japan).

\section{Data quality assurance}

To ensure the quality of the data, good laboratory practice was followed in all stages of the work. All reagents used were checked for their expiry date and used according to the manufacturer's instructions.

\section{Data analysis}

Data were entered into Epi-info version 3.5.1 and then exported to SPSS version 20 (SPSS Inc., Chicago, IL, USA). Independent $t$-test was performed to determine significant differences between type 2 diabetics and nondiabetics in terms of FBG, weight, body mass index (BMI), waist-to-hip ratio (WHR), blood pressure (systolic and diastolic), ferritin, and trace metal elements. Levene's test for homogeneity of variances was calculated in order to accept or reject the null hypnosis. To determine possible relationships between parameters, Pearson's correlation coefficient $(r)$ was used. All variables with $p$-value less than 0.05 were considered as statistically significant.

\section{Reference ranges}

The reference ranges used to determine serum ferritin, trace metal elements, FBG, and CRP were obtained from different literature ${ }^{13-15}$ and presented as follows: $\mathrm{Zn}^{+2}(75-120 \mu \mathrm{g} / \mathrm{dL})$, $\mathrm{Mg}^{+2}(1.6-2.6 \mathrm{mg} / \mathrm{dL}), \mathrm{Cr}^{+3}(0.05-0.5 \mu \mathrm{g} / \mathrm{L}), \mathrm{Fe}^{+3}$ for male $(65-165 \mu \mathrm{g} / \mathrm{dL})$ and for female $(50-70 \mu \mathrm{g} / \mathrm{dL})$, ferritin for male $(20-250 \mu \mathrm{g} / \mathrm{L})$ and for female $(10-120 \mu \mathrm{g} / \mathrm{L}), \mathrm{Cu}^{+2}$ $(70-140 \mu \mathrm{g} / \mathrm{dL}), \mathrm{Mn}^{+2}(0.4-1.1 \mu \mathrm{g} / \mathrm{L}), \mathrm{Ca}^{+2}(4.64-5.28 \mathrm{mg} /$ $\mathrm{dL}), \mathrm{PO}_{4}^{-3}(2.5-4.5 \mathrm{mg} / \mathrm{dL}), \mathrm{FBG}(\leq 126 \mathrm{mg} / \mathrm{dL})$, and CRP $(\leq 10 \mathrm{mg} / \mathrm{dL})$.

\section{Results}

It is common scientific knowledge that there is a strong association between type 2 diabetes and increased FBG, BMI, waist circumference, and WHR. The results of the present study are consistent with this fact. As shown in Table 1, FBG, BMI, WHR, and systolic and diastolic blood pressures in type 2 diabetes patients were significantly $(p<0.0001)$ higher than in nondiabetes study subjects.

Study subjects with T2DM had a significant $(p<0.0001)$ lower serum $\mathrm{Zn}^{+2}, \mathrm{Mg}^{+2}, \mathrm{Cr}^{+3}, \mathrm{Fe}^{+3}$, and ferritin concentrations than nondiabetics (Table 2). In contrast, the serum $\mathrm{Cu}^{+2}$ concentration was significantly $(p<0.0001)$ higher in T2DM patients than in nondiabetics. Significant differences were not observed in both study subjects with regard to the serum concentration of $\mathrm{Mn}^{+2}, \mathrm{Ca}^{+2}$, and $\mathrm{Po}_{4}^{-3}$.

Tables 3 and 4 show the mean differences in age, BMI, WHR, systolic and diastolic blood pressures, fasting plasma glucose, ferritin, and trace metal elements among T2DM study subjects who had been taking hypoglycemic agents and insulin as treatment options.

WHR was significantly higher $(p<0.0001)$ among oral hypoglycemic agents taking T2DM study subjects than

Table I Clinical characterization of the diabetes and nondiabetes study subjects

\begin{tabular}{|c|c|c|c|c|}
\hline \multirow{2}{*}{$\begin{array}{l}\text { Tested } \\
\text { parameters }\end{array}$} & \multicolumn{2}{|c|}{ Study subjects } & \multirow[t]{2}{*}{$t$-test } & \multirow[t]{2}{*}{$p$-value } \\
\hline & $\begin{array}{l}\text { Diabetes } \\
(n=2 \mid 4)\end{array}$ & $\begin{array}{l}\text { Nondiabetes } \\
(n=2 \mid 4)\end{array}$ & & \\
\hline ge (years) & $43.18 \pm 12.68$ & $43.18 \pm 12.68$ & -0.37 & 0.712 \\
\hline $\begin{array}{l}\text { Duration with } \\
\text { diabetes (years) }\end{array}$ & $5.83 \pm 3.11$ & - & - & - \\
\hline $\begin{array}{l}\text { Fasting blood } \\
\text { glucose (mg/dL) }\end{array}$ & $162.16 \pm 29.53$ & $78.56 \pm 10.73$ & 38.92 & $<0.000 I^{*}$ \\
\hline $\begin{array}{l}\text { Body mass index } \\
\left(\mathrm{kg} / \mathrm{m}^{2}\right)\end{array}$ & $26.15 \pm 4.53$ & $23.94 \pm 4.68$ & 4.93 & $<0.000 I^{*}$ \\
\hline $\begin{array}{l}\text { Waist and hip } \\
\text { circumference }(\mathrm{cm})\end{array}$ & $1.43 \pm 0.56$ & $1.04 \pm 0.37$ & 8.52 & $<0.000 I^{*}$ \\
\hline $\begin{array}{l}\text { Systolic blood } \\
\text { pressure }(\mathrm{mmHg})\end{array}$ & $134.2 \pm 22.62$ & $122.04 \pm 18.76$ & 6.05 & $<0.000$ I $^{*}$ \\
\hline $\begin{array}{l}\text { Diastolic blood } \\
\text { pressure }(\mathrm{mmHg})\end{array}$ & $88.36 \pm 13.23$ & $84.14 \pm 13.92$ & 3.21 & $<0.000 I^{*}$ \\
\hline
\end{tabular}

Notes: Data were presented as mean (standard deviation). ${ }^{*} p<0.05$ indicates a significant difference. Independent $t$-test. 
Table 2 Levels of ferritin and metal elements in the serum of diabetes and nondiabetes study subjects

\begin{tabular}{|c|c|c|c|c|}
\hline \multirow{2}{*}{$\begin{array}{l}\text { Tested } \\
\text { parameters }\end{array}$} & \multicolumn{2}{|c|}{ Study subjects } & \multirow[t]{2}{*}{$t$-test } & \multirow[t]{2}{*}{$p$-value } \\
\hline & $\begin{array}{l}\text { Diabetes } \\
(n=2 \mid 4)\end{array}$ & $\begin{array}{l}\text { Nondiabetes } \\
(n=2 \mid 4)\end{array}$ & & \\
\hline $\mathrm{Zn}^{+2}(\mu \mathrm{g} / \mathrm{dL})$ & $46.26 \pm 21.03$ & $72.4 I \pm 2 I .47$ & -12.72 & $<0.000 I^{*}$ \\
\hline $\mathrm{Mg}^{+2}(\mathrm{mg} / \mathrm{dL})$ & $0.86 \pm 0.88$ & $1.82 \pm 0.76$ & -11.95 & $<0.000 I^{*}$ \\
\hline $\mathrm{Cr}^{+3}(\mu \mathrm{g} / \mathrm{L})$ & $0.82 \pm 1.42$ & $0.35 \pm 0.43$ & 4.57 & $<0.000 I^{*}$ \\
\hline $\mathrm{Fe}^{+3}(\mu \mathrm{g} / \mathrm{dL})$ & $55.95 \pm 20.99$ & $71.19 \pm 25.72$ & -6.70 & $<0.000 I^{*}$ \\
\hline Ferritin $(\mu g / L)$ & $24.96 \pm 18.60$ & $34.99 \pm 18.53$ & -5.58 & $<0.000 I^{*}$ \\
\hline $\mathrm{Cu}^{+2}(\mu \mathrm{g} / \mathrm{dL})$ & $79.61 \pm 27.38$ & $71.67 \pm 22.38$ & 3.28 & $<0.000 I^{*}$ \\
\hline $\mathrm{Mn}^{+2}(\mu \mathrm{g} / \mathrm{L})$ & $0.65 \pm 0.91$ & $0.66 \pm 0.45$ & -0.18 & 0.853 \\
\hline $\mathrm{Ca}^{+2}(\mathrm{mg} / \mathrm{dL})$ & $4.28 \pm 1.19$ & $4.36 \pm 0.56$ & -0.83 & 0.407 \\
\hline $\mathrm{Po}_{4}^{-3}(\mathrm{mg} / \mathrm{dL})$ & $2.16 \pm 0.98$ & $2.27 \pm 0.56$ & -1.40 & 0.162 \\
\hline
\end{tabular}

Notes: Data were presented as mean (standard deviation). ${ }^{*} p<0.05$ indicates a significant difference. Independent $t$-test.

Table 3 Clinical characteristics among oral hypoglycemic agent and injectable insulin users of diabetes study subjects

\begin{tabular}{|c|c|c|c|c|}
\hline \multirow{2}{*}{$\begin{array}{l}\text { Tested } \\
\text { parameters }\end{array}$} & \multicolumn{2}{|c|}{ Diabetes treatment options } & \multirow[t]{2}{*}{$t$-test } & \multirow[t]{2}{*}{$p$-value } \\
\hline & $\begin{array}{l}\text { Oral } \\
\text { hypoglycemic } \\
\text { agents }(n=161)\end{array}$ & $\begin{array}{l}\text { Injectable } \\
\text { insulin } \\
(n=53)\end{array}$ & & \\
\hline Age (years) & $42.57 \pm 13.4$ & $44.11 \pm 9.9$ & -0.894 & 0.373 \\
\hline $\begin{array}{l}\text { Fasting blood } \\
\text { glucose }(\mathrm{mg} / \mathrm{dL})\end{array}$ & $159.88 \pm 27.4$ & $169.07 \pm 34.5$ & -1.761 & 0.82 \\
\hline $\begin{array}{l}\text { Body mass index } \\
\left(\mathrm{kg} / \mathrm{m}^{2}\right)\end{array}$ & $26.3 \pm 4.86$ & $25.65 \pm 3.35$ & 1.084 & 0.281 \\
\hline $\begin{array}{l}\text { Waist and hip } \\
\text { circumference } \\
(\mathrm{cm})\end{array}$ & $1.5 \pm 0.59$ & $1.22 \pm 0.43$ & 3.778 & $<0.000 I^{*}$ \\
\hline $\begin{array}{l}\text { Systolic blood } \\
\text { pressure }(\mathrm{mmHg})\end{array}$ & $134.3 \pm 24 . \mid$ & $133.9 \pm 17.5$ & 0.105 & 0.928 \\
\hline $\begin{array}{l}\text { Diastolic blood } \\
\text { pressure }(\mathrm{mmHg})\end{array}$ & $87.2 \pm 13.2$ & $91.8 \pm 12.8$ & -2.255 & 0.025 \\
\hline
\end{tabular}

Notes: Data were presented as mean (standard deviation). ${ }^{*} p<0.05$ indicates a significant difference. Independent $t$-test.

Table 4 Levels of serum ferritin and metal elements among oral hypoglycemic agent and injectable insulin users of diabetes study subjects

\begin{tabular}{|c|c|c|c|c|}
\hline \multirow{2}{*}{$\begin{array}{l}\text { Tested } \\
\text { parameters }\end{array}$} & \multicolumn{2}{|c|}{ Diabetes treatment options } & \multirow[t]{2}{*}{ t-test } & \multirow[t]{2}{*}{$p$-value } \\
\hline & $\begin{array}{l}\text { Oral } \\
\text { hypoglycemic } \\
\text { agents }(n=161)\end{array}$ & $\begin{array}{l}\text { Injectable } \\
\text { insulin } \\
(n=53)\end{array}$ & & \\
\hline $\mathrm{Zn}^{+2}(\mu \mathrm{g} / \mathrm{dL})$ & $45.8 \pm 20.84$ & $47.6 \pm 21.76$ & -0.550 & 0.583 \\
\hline $\mathrm{Mg}^{+2}(\mathrm{mg} / \mathrm{dL})$ & $0.85 \pm 0.89$ & $0.89 \pm 0.89$ & -0.216 & 0.829 \\
\hline $\mathrm{Cr}^{+3}(\mu \mathrm{g} / \mathrm{L})$ & $0.83 \pm 1.45$ & $0.78 \pm 1.34$ & 0.229 & 0.819 \\
\hline $\mathrm{Fe}^{+3}(\mu \mathrm{g} / \mathrm{dL})$ & $57.79 \pm 20.49$ & $50.37 \pm 21.71$ & 2.252 & $<0.025^{*}$ \\
\hline Ferritin $(\mu g / L)$ & $26.72 \pm 19.70$ & $19.62 \pm 13.58$ & 2.439 & $<0.016^{*}$ \\
\hline $\mathrm{Cu}^{+2}(\mu \mathrm{g} / \mathrm{dL})$ & $81.28 \pm 29.06$ & $74.52 \pm 20.88$ & 1.56 & 1.119 \\
\hline $\mathrm{Mn}^{+2}(\mu \mathrm{g} / \mathrm{L})$ & $0.75 \pm 0.96$ & $0.35 \pm 0.6 \mathrm{I}$ & 3.52 & $0.001 *$ \\
\hline $\mathrm{Ca}^{+2}(\mathrm{mg} / \mathrm{dL})$ & $4.28 \pm 1.28$ & $4.3 \pm 0.86$ & -0.156 & 0.877 \\
\hline $\mathrm{Po}_{4}^{-3}(\mathrm{mg} / \mathrm{dL})$ & $2.11 \pm 1.07$ & $2.34 \pm 0.65$ & -1.88 & 0.062 \\
\hline
\end{tabular}

Notes: Data were presented as mean (standard deviation). ${ }^{*} p<0.05$ indicates a significant difference. Independent $t$-test. insulin. Whereas diastolic blood pressure was significantly higher $(p<0.05)$ among insulin taking T2DM study subjects than oral hypoglycemic agents (Table 3 ). Serum iron, ferritin, and manganese levels were significantly higher $(p<0.05)$ among T2DM study subjects, who had been taking oral hypoglycemic agent as a treatment option than insulin (Table 4).

Pearson bivariate correlation analysis was applied to investigate the interrelationships of ferritin and trace metal elements and with clinically useful parameters, such as BMI, WHR, and FBG in the serum of T2DM study subjects. Thus, significant positive correlation was detected between serum $\mathrm{Zn}^{+2}$ with serum $\mathrm{Mg}^{+2}(r=0.738), \mathrm{Cr}^{+3}(r=0.233)$, $\mathrm{Ca}^{+2}(r=0.238)$, and $\mathrm{Po}_{4}^{-3}(r=0.222)$; serum $\mathrm{Mg}^{+2}$ with $\mathrm{Zn}^{+2}$ ( $r=0.738), \mathrm{Cr}^{+3}(r=0.358)$, and $\mathrm{Po}_{4}{ }^{-3}(r=0.209$; Table 5). Serum $\mathrm{Zn}^{+2}$ also significantly and negatively correlated with BMI $(r=-0.331)$, WHR $(r=-0.340)$, and FBG $(r=-0.186)$. Likewise, serum $\mathrm{Mg}^{+2}$ and $\mathrm{Po}_{4}^{-3}$ are significantly and negatively correlated with BMI, WHR, and FBG (Table 6).

\section{Discussion}

Trace metal elements are very important elements for cellular metabolic reactions, and their deviation from the normal physiologic range may put adverse effect on biological process. ${ }^{16,17}$ In our finding, the serum concentration of zinc, magnesium, and chromium in patients with T2DM was significantly lower than the nondiabetes study subjects. The finding is in harmony with previous studies. ${ }^{18-25}$ However, some studies showed insignificant difference of serum zinc and magnesium among T2DM patients and nondiabetes study subjects, ${ }^{26-28}$ which are inconsistent with the current finding. Zinc and magnesium are essential trace metal elements crucial for the function of more than 300 enzymes. ${ }^{29-33}$ The trivalent chromium is often required for the maintenance of normal glucose ${ }^{34}$ and fat metabolism. ${ }^{35}$ Chromium stimulates insulin signaling pathway and increases glucose transporter protein translocation in muscle cells. ${ }^{1}$ However, zinc, magnesium, and chromium deficiency often leads to insulin resistance. ${ }^{29-37}$ The exact physiologic mechanism of zinc, magnesium, and chromium deficiency among T2DM patients is unknown, but high urinary loss has been observed as a result of the osmotic effect of glycosuria. ${ }^{20,25,33}$ Increased serum iron and ferritin concentrations have been reported to be associated with insulin resistance. ${ }^{38-45}$ Our findings on serum iron and ferritin concentration were inconsistent with the previous findings. ${ }^{38-45}$ Iron plays an essential role as a cofactor for fuel oxidation and electron transport, but it also has the potential to cause oxidative damage if not 
Table 5 Pearson bivariate correlation analysis on serum ferritin and metal elements among diabetes study subjects

\begin{tabular}{|c|c|c|c|c|c|c|c|c|c|}
\hline Tested parameters & $\begin{array}{l}Z^{+2} \\
(\mu g / d L)\end{array}$ & $\begin{array}{l}\mathrm{Mg}^{+2} \\
(\mathrm{mg} / \mathrm{dL})\end{array}$ & $\begin{array}{l}\mathrm{Cr}^{+3} \\
(\mu \mathrm{g} / \mathrm{L})\end{array}$ & $\begin{array}{l}\mathrm{Fe}^{+3} \\
(\mu \mathrm{g} / \mathrm{dL})\end{array}$ & $\begin{array}{l}\text { Ferritin } \\
(\mu \mathrm{g} / \mathrm{L})\end{array}$ & $\begin{array}{l}\mathrm{Cu}^{+2} \\
(\mu \mathrm{g} / \mathrm{dL})\end{array}$ & $\begin{array}{l}M n^{+2} \\
(\mu g / L)\end{array}$ & $\begin{array}{l}\mathrm{Ca}^{+2} \\
(\mathrm{mg} / \mathrm{dL})\end{array}$ & $\begin{array}{l}\mathrm{Po}_{4}^{+3} \\
(\mathrm{mg} / \mathrm{dL})\end{array}$ \\
\hline \multicolumn{10}{|l|}{$\mathrm{Zn}^{+2}(\mu \mathrm{g} / \mathrm{dL})$} \\
\hline$r$ & 1 & 0.738 & 0.233 & 0.062 & -0.032 & -0.296 & 0.039 & 0.238 & 0.222 \\
\hline$p$-Value & - & $<0.0001 * *$ & $<0.001 * *$ & 0.367 & 0.645 & $<0.000$ I** & 0.572 & $<0.0001 * *$ & $<0.001 * *$ \\
\hline \multicolumn{10}{|l|}{$\mathrm{Mg}^{+2}(\mathrm{mg} / \mathrm{dL})$} \\
\hline$r$ & 0.738 & 1 & 0.358 & -0.103 & -0.042 & -0.171 & 0.079 & 0.157 & 0.209 \\
\hline$p$-Value & $<0.000 I^{* *}$ & - & $<0.000 I^{* *}$ & 0.134 & 0.544 & $0.012^{* *}$ & 0.250 & 0.022 & $0.002 * *$ \\
\hline \multicolumn{10}{|l|}{$\mathrm{Cr}^{+3}(\mu \mathrm{g} / \mathrm{L})$} \\
\hline$r$ & 0.233 & 0.358 & 1 & 0.01 & 0.031 & -0.03 & 0.068 & 0.211 & 0.298 \\
\hline$p$-Value & $<0.00 I^{* *}$ & $<0.000 I^{* * *}$ & - & 0.885 & 0.653 & 0.661 & 0.321 & $0.002^{*}$ & $<0.000 I^{* *}$ \\
\hline \multicolumn{10}{|l|}{$\mathrm{Fe}^{+3}(\mu \mathrm{g} / \mathrm{dL})$} \\
\hline$r$ & 0.062 & -0.103 & 0.010 & 1 & 0.665 & 0.189 & 0.065 & 0.020 & -0.058 \\
\hline$p$-Value & 0.367 & 0.134 & 0.885 & - & $<0.0001 * *$ & $0.0001 * *$ & 0.343 & 0.772 & 0.402 \\
\hline \multicolumn{10}{|l|}{ Ferritin ( $\mu \mathrm{g} / \mathrm{L})$} \\
\hline$r$ & -0.032 & -0.042 & 0.031 & 0.665 & 1 & 0.327 & 0.085 & 0.024 & 0.006 \\
\hline$p$-Value & 0.645 & 0.544 & 0.653 & $<0.000 I^{* *}$ & - & $<0.0001 * *$ & 0.218 & 0.732 & 0.927 \\
\hline \multicolumn{10}{|l|}{$\mathrm{Cu}^{+2}(\mu \mathrm{g} / \mathrm{dL})$} \\
\hline$r$ & -0.296 & $-0.17 \mid$ & -0.03 & 0.189 & 0.327 & 1 & 0.275 & -0.138 & -0.087 \\
\hline$p$-Value & $<0.000 I^{* *}$ & $0.012^{* *}$ & $0.66 \mathrm{I}$ & $<0.000 I^{* *}$ & $<0.0001 * *$ & - & $<0.000 I^{* *}$ & 0.044 & 0.205 \\
\hline \multicolumn{10}{|l|}{$\mathrm{Mn}^{+2}(\mu \mathrm{g} / \mathrm{L})$} \\
\hline$r$ & 0.039 & 0.079 & 0.068 & 0.065 & 0.085 & 0.275 & I & -0.144 & -0.090 \\
\hline$p$-Value & 0.572 & 0.250 & 0.321 & 0.343 & 0.218 & $<0.000$ I** & - & 0.036 & 0.192 \\
\hline \multicolumn{10}{|l|}{$\mathrm{Ca}^{+2}(\mathrm{mg} / \mathrm{dL})$} \\
\hline$r$ & 0.238 & 0.157 & 0.211 & 0.020 & 0.024 & -0.138 & -0.144 & 1 & 0.527 \\
\hline$p$-Value & $<0.000 I^{* *}$ & 0.022 & $0.002^{* *}$ & 0.772 & 0.732 & 0.044 & 0.036 & - & $<0.000 I^{* *}$ \\
\hline \multicolumn{10}{|l|}{$\mathrm{Po}_{4}^{+3}(\mathrm{mg} / \mathrm{dL})$} \\
\hline$r$ & 0.222 & 0.209 & 0.298 & -0.058 & 0.006 & -0.087 & -0.090 & 0.527 & 1 \\
\hline$p$-Value & $<0.00 I^{* *}$ & $0.002^{* *}$ & $<0.000 I^{* *}$ & 0.402 & 0.927 & 0.205 & 0.192 & $<0.0001 * *$ & - \\
\hline
\end{tabular}

Note: **Pearson correlation is significant at the 0.01 level (two tailed).

Table 6 Pearson bivariate correlation analysis on serum ferritin and metal elements with body mass index, waist-to-hip ratio, and fasting blood glucose among diabetes study subjects

\begin{tabular}{|c|c|c|c|c|c|c|}
\hline \multirow[t]{2}{*}{ Tested parameters } & \multicolumn{2}{|c|}{ Body mass index $\left(\mathrm{kg} / \mathrm{m}^{2}\right)$} & \multicolumn{2}{|c|}{ Waist-to-hip ratio $(\mathrm{cm})$} & \multicolumn{2}{|c|}{ Fasting blood glucose $(\mathrm{mg} / \mathrm{dL}$} \\
\hline & $r$ & $p$-value & $r$ & $p$-value & $r$ & $p$-value \\
\hline $\mathrm{Zn}^{+2}(\mu \mathrm{g} / \mathrm{dL})$ & -0.331 & $<0.000 I^{* *}$ & -0.340 & $<0.000 I^{* * *}$ & -0.186 & $0.006 * *$ \\
\hline $\mathrm{Mg}^{+2}(\mathrm{mg} / \mathrm{dL})$ & -0.254 & $<0.000 I^{* *}$ & -0.199 & $0.003^{*}$ & -0.136 & $<0.000 I^{* * *}$ \\
\hline $\mathrm{Cr}^{+3}(\mu \mathrm{g} / \mathrm{L})$ & -0.121 & 0.077 & 0.002 & 0.974 & -0.023 & 0.734 \\
\hline $\mathrm{Fe}^{+3}(\mu \mathrm{g} / \mathrm{dL})$ & -0.099 & 0.148 & -0.029 & 0.672 & -0.111 & 0.106 \\
\hline Ferritin $(\mu g / L)$ & -0.058 & 0.396 & 0.053 & 0.443 & -0.100 & 0.144 \\
\hline $\mathrm{Cu}^{+2}(\mu g / d L)$ & 0.140 & 0.04 & 0.327 & $<0.000 I^{* *}$ & 0.057 & 0.404 \\
\hline $\mathrm{Mn}^{+2}(\mu \mathrm{g} / \mathrm{L})$ & 0.021 & 0.755 & 0.282 & $<0.000 I^{* *}$ & -0.008 & 0.912 \\
\hline $\mathrm{Ca}^{+2}(\mathrm{mg} / \mathrm{dL})$ & -0.335 & $<0.000 I^{* *}$ & -0.372 & $<0.0001 * *$ & -0.141 & 0.040 \\
\hline $\mathrm{Po}_{4}^{-3}(\mathrm{mg} / \mathrm{dL})$ & -0.380 & $<0.000 I^{* *}$ & -0.309 & $<0.000 I^{* *}$ & -0.159 & $<0.0001 * *$ \\
\hline
\end{tabular}

Notes: Data were presented as mean (standard deviation). **Pearson correlation is significant at the 0.01 level (two tailed).

carefully regulated and found in excess in the tissue. ${ }^{46}$ Ferritin is a ubiquitous intracellular protein commonly utilized as a marker of body iron stores. ${ }^{41,43,44}$ Ferritin can release iron if the blood has a low iron concentration and store excess iron if the blood and tissues have a high iron concentration. ${ }^{47,48}$ The imbalance of blood iron and ferritin in diabetes is mysterious. ${ }^{49}$ But, inadequate dietary intake, asymptomatic illness, and poor absorption rate often associated with the decrease of iron and ferritin. Different studies have been showing the increase of serum copper in T2DM patients, while urinary excretion of copper is high. $16,18,21,23,26,27,50,51$ Our study has shown significant elevation of serum copper concentration in patients with type 2 diabetes than nondiabetes. Again our finding on serum copper disagree with some other findings 
as well. ${ }^{52}$ Copper is considered as a powerful enzyme catalyst and its deficiency results in glucose intolerance, hypercholesterolemia, and atherosclerosis. ${ }^{53}$ Inconsistent with others findings, ${ }^{54-57}$ significant difference was not observed in both T2DM patients and nondiabetes regarding serum manganese. Manganese is an important constituent of some enzymes, such as pyruvate carboxylase and arginase. ${ }^{1}$ Normal levels of manganese are involved in the synthesis and secretion of insulin. ${ }^{54-57}$ Generally, studies are not adequate on calcium and phosphorus in the blood sample of both diabetes and nondiabetes study subjects. But, data from animal models of diabetes and from studying patients with diabetes reveal increased intracellular calcium level in most tissues. ${ }^{58,59}$ However, in the current study, significant difference was not observed in serum calcium and phosphorus in both type 2 diabetes and nondiabetes study subjects. In the current study, we have also compared some clinical parameters, such as BMI, WHR, systolic and diastolic blood pressures, FBG, serum trace metal elements, and ferritin level among oral hypoglycemic agent and injectable insulin users of T2DM study subjects. Thus, WHR, diastolic blood pressure, serum iron, ferritin, and manganese level were significantly higher among oral hypoglycemic agent users of T2DM study subjects than injectable insulin users. Although we do not know the exact mechanism for such differences, usually injectable insulin is much more effective to achieve near normal glycemic control as early as possible in the course of the disease. ${ }^{60}$

\section{Limitation of the study}

We did not measure tissue and urinary trace metal elements, glycated hemoglobin, and postprandial insulin level of the study subjects. Moreover, the study was cross-sectional and parameters were measured once. Thus, relationship between the measured parameters and the T2DM may not be truly associated. Thus, we would like to recommend the researchers to study more about the association of ferritin, trace metal elements, and T2DM in a cohort study with larger number of study subjects.

\section{Conclusion}

In general, the current study shows decrease of serum zinc, magnesium, chromium, iron, ferritin and increase of serum copper concentration in T2DM study subjects than in nondiabetes mellitus study subjects. But, serum manganese, calcium, and phosphorus did not show significant differences in both the study subjects. Additionally, WHR, serum iron, ferritin, and manganese were significantly higher among oral hypoglycemic agent users of T2DM study subjects.
Moreover, significant positive and negative correlation has been seen between serum zinc with magnesium, chromium, copper, calcium, and phosphorus and magnesium with chromium, copper, calcium, and phosphorus among T2DM study subjects (details are described in Table 5). Zinc, magnesium, and phosphorus also negatively correlated with BMI, WHR, and FBG level (details are described in Table 6). We believe that poor glycemic control, poor adherences toward insulin and oral hypoglycemic agents, high urinary clearance rate, asymptomatic disease, oxidative stress, and other health and health-related factors could be contributing factors for the existing disturbed trace metal elements among T2DM patients. Thus, following the possible preventable strategies (lifestyle modification and good adherences to medication) may reduce the complication of diabetes and trace metal elements in T2DM patients.

\section{Acknowledgments}

We would like to express our esteemed gratitude to our study participants and data collectors for their cooperation. We also would like to thank Jimma University College of Health Sciences for funding and Jimma University Specialized Hospital for allowing us to conduct the research in the diabetic clinic.

\section{Disclosure}

The authors report no conflicts of interest in this work.

\section{References}

1. Khan AR, Awan FR. Metals in the pathogenesis of type 2 diabetes. J Diabetes Metab Disord. 2014;13(1):16.

2. Badran M, Morsy R, Soliman H, Elnimr T. Assessment of trace elements levels in patients with type 2 diabetes using multivariate statistical analysis. J Trace Elem Med Biol. 2016;33:114-119.

3. Wiernsperger N, Rapin J. Trace elements in glucometabolic disorders: an update. Diabetol Metab Syndr. 2010;2(1):70.

4. American Diabetes Association. Diagnosis and classification of diabetes mellitus. Diabetes Care. 2006;29(Suppl 1):S43-S48.

5. Ginter E, Simko V. Global prevalence and future of diabetes mellitus. In: Diabetes. New York, NY: Springer; 2013:35-41.

6. International Diabetes Federation: Diabetes in Ethiopia [updated 2015]. Available from: http://www.idf.org/membership/afr/ethiopia. Accessed August 21, 2016.

7. Flores CR, Puga MP, Wrobel K, Sevilla ME, Wrobel K. Trace elements status in diabetes mellitus type 2: possible role of the interaction between molybdenum and copper in the progress of typical complications. Diabetes Res Clin Pract. 2011;91(3):333-341.

8. Idänpään-Heikkilä JE. Ethical principles for the guidance of physicians in medical research: the Declaration of Helsinki. Bull World Health Organ. 2001;79(4):279.

9. Hall GC, Sauer B, Bourke A, Brown JS, Reynolds MW, Casale RL. Guidelines for good database selection and use in pharmacoepidemiology research. Pharmacoepidemiol Drug Saf. 2012;21(1):1-10.

10. Yahaya MI, Shehu A, Dabai FG. Efficiency of extraction of trace metals from blood samples using wet digestion and microwave digestion techniques. J Appl Sci Environ Manag. 2013;17(3): 365-369. 
11. Li G, Brockman JD, Lin SW, Abnet CC, Schell LA, Robertson JD. Measurement of the trace elements $\mathrm{Cu}, \mathrm{Zn}, \mathrm{Fe}$, and $\mathrm{Mg}$ and the ultratrace elements $\mathrm{Cd}, \mathrm{Co}, \mathrm{Mn}$, and $\mathrm{Pb}$ in limited quantity human plasma and serum samples by inductively coupled plasma-mass spectrometry. Am J Analyt Chem. 2012;3(9):646.

12. Badran M, Ismail A, Morsy R, Elnimr T. Critical evaluating for five digestion methods using ICP-MS. J Pharm Sci Innovation. 2014;3:30-34.

13. Bogden JD, Klevay LM. Clinical Nutrition of the Essential Trace Elements and Minerals: The Guide for Health Professionals. Totowa, NJ: Humana Press; 2000.

14. Iyengar V, Woittiez J. Trace elements in human clinical specimens: evaluation of literature data to identify reference values. Clin Chem. 1988;34(3):474-481.

15. Kratz A, Pesce MA, Fink DJ. Appendix: laboratory values of clinical importance. Harrison's principles of internal medicine, 19e. Available from: http://www. accessmedicine. com/content. aspx. 2012. Accessed August 10, 2015

16. Khan FA, Al Jameil N, Arjumand S, et al. Comparative study of serum copper, iron, magnesium, and zinc in type 2 diabetes-associated proteinuria. Biol Trace Elem Res. 2015;168(2):321-329.

17. Basaki M, Saeb M, Nazifi S, Shamsaei HA. Zinc, copper, iron, and chromium concentrations in young patients with type 2 diabetes mellitus. Biol Trace Elem Res. 2012;148(2):161-164.

18. Oyedeji SO, Adesina AA, Oke OT, Tijani YO. Evaluation of essential trace metals in female type 2 diabetes mellitus patients in Nigerian population. Afr J Biotechnol. 2014;13(18):1910-1914.

19. Hussain F, Maan MA, Sheikh MA, Nawaz H, Jamil A. Trace elements status in type 2 diabetes. Bangladesh J Med Sci. 2009;8(3):52.

20. Ferdousi S, Mollah FH, Mia MA. Serum levels of zinc and magnesium in newly diagnosed type-2 diabetic subjects. Bangladesh JMed Biochem. 2013;3(2):46-49.

21. Viktorínová A, Tošerová E, Križko M, Duračková Z. Altered metabolism of copper, zinc, and magnesium is associated with increased levels of glycated hemoglobin in patients with diabetes mellitus. Metabolism. 2009;58(10): 1477-1482.

22. Onah CE, Meludu SC, Dioka CE, Amah UK, Okwara JE, Osuji CU. Evaluation of selected trace elements in male type 2 diabetic patients in Nnewi, south eastern Nigeria. J Health Spec. 2013;1(3):129.

23. Abou-Seif MA, Youssef AA. Evaluation of some biochemical changes in diabetic patients. Clin Chim Acta. 2004;346(2):161-170.

24. Ghosh D, Bhattacharya B, Mukherjee B, Manna B, Sinha M, Chowdhury J, Chowdhury S. Role of chromium supplementation in Indians with type 2 diabetes mellitus. J Nutr Biochem. 2002;13(11):690-697.

25. Ding W, Chai Z, Duan P, Feng W, Qian Q. Serum and urine chromium concentrations in elderly diabetics. Biol Trace Elem Res. 1998;63(3):231-237.

26. Walter RM, Uriu-Hare JY, Olin KL, Oster MH, Anawalt BD, Critchfield JW, Keen CL. Copper, zinc, manganese, and magnesium status and complications of diabetes mellitus. Diabetes Care. 1991;14(11):1050-1056.

27. Zargar AH, Shah NA, Masoodi SR, et al. Copper, zinc, and magnesium levels in non-insulin dependent diabetes mellitus. Postgrad Med J. 1998;74(877):665-668.

28. Rusu ML, Marutoiu C, Rusu LD, Marutoiu OF, Hotoleanu C, Poanta L. Testing of magnesium, zinc and copper blood levels in diabetes mellitus patients. Int $J$ Acta Universitatis Cibiniensis Seria F Chemia. 2005;8:61-63.

29. Jansen J, Karges W, Rink L. Zinc and diabetes-clinical links and molecular mechanisms. J Nutr Biochem. 2009;20(6):399-417.

30. Sales $\mathrm{CH}$, Pedrosa LD. Magnesium and diabetes mellitus: their relation. Clin Nutr. 2006;25(4):554-562.

31. de Lourdes Lima M, Cruz T, Rodrigues LE, et al. Serum and intracellular magnesium deficiency in patients with metabolic syndrome: evidences for its relation to insulin resistance. Diabetes Res Clin Pract. 2009;83(2):257-262.

32. Masood N, Baloch GH, Ghori RA, Memon IA, Memon MA, Memon MS. Serum zinc and magnesium in type-2 diabetic patients. J Coll Physicians Surg Pak. 2009;19(8):483-486.
33. Praveeena S, PaSula S, Sameera K. Trace elements in diabetes mellitus. J Clin Diagn Res. 2013;7(9):1863-1865.

34. O'Connell BS. Select vitamins and minerals in the management of diabetes. Diabetes Spectr. 2001;14(3):133-148.

35. Guerrero-Romero F, Rodríguez-Morán M. Complementary therapies for diabetes: the case for chromium, magnesium, and antioxidants. Arch Med Res. 2005;36(3):250-257.

36. Doddigarla Z, Parwez I, Ahmad J. Correlation of serum chromium, zinc, magnesium and SOD levels with HbA1c in type 2 diabetes: a cross sectional analysis. Diabetes Metab Syndr. 2016;10(1 Suppl 1):S126-S129.

37. Althuis MD, Jordan NE, Ludington EA, Wittes JT. Glucose and insulin responses to dietary chromium supplements: a meta-analysis. Am J Clin Nutr. 2002;76(1):148-155.

38. Kim CH, Kim HK, Bae SJ, Park JY, Lee KU. Association of elevated serum ferritin concentration with insulin resistance and impaired glucose metabolism in Korean men and women. Metabolism. 2011;60(3):414-420.

39. Cheung CL, Cheung TT, Lam KS, Cheung BM. High ferritin and low transferrin saturation are associated with pre-diabetes among a national representative sample of US adults. Clin Nutr. 2013;32(6):1055-1060.

40. Rajpathak SN, Crandall JP, Wylie-Rosett J, Kabat GC, Rohan TE, Hu FB The role of iron in type 2 diabetes in humans. Biochim Biophysi Acta. 2009;1790(7):671-681.

41. Mojiminiyi OA, Marouf R, Abdella NA. Body iron stores in relation to the metabolic syndrome, glycemic control and complications in female patients with type 2 diabetes. Nutr Metab Cardiovasc Dis. 2008;18(8):559-566.

42. Orban E, Schwab S, Thorand B, Huth C. Association of iron indices and type 2 diabetes: a meta-analysis of observational studies. Diabetes Metab Res Rev. 2014;30(5):372-394.

43. Andrews M, Soto N, Arredondo-Olguín M. Association between ferritin and hepcidin levels and inflammatory status in patients with type 2 diabetes mellitus and obesity. Nutrition. 2015;31(1):51-57.

44. Fernández-Real JM, López-Bermejo A, Ricart W. Cross-talk between iron metabolism and diabetes. Diabetes. 2002;51(8):2348-2354.

45. Yiqiang $Z$, Zheng T, Jinming Y. Serum ferritin, diabetes, diabetes control, and insulin resistance. Acta Diabetol. 2014;51:991-998.

46. Simcox JA, Mitchell TC, Gao Y, et al. Dietary iron controls circadian hepatic glucose metabolism through heme synthesis. Diabetes. 2015;64(4):1108-1119.

47. Raghavani PH, Sirajwala HB. Serum ferritin level in patients with type-2 diabetes mellitus. Intern J Biomed Adv Res. 2014;5(6):272-274.

48. Lee BK, Kim Y, Kim YI. Association of serum ferritin with metabolic syndrome and diabetes mellitus in the South Korean general population according to the Korean National Health and Nutrition Examination Survey 2008. Metabolism. 2011;60(10):1416-1424.

49. Swaminathan S, Fonseca VA, Alam MG, Shah SV. The role of iron in diabetes and its complications. Diabetes Care. 2007;30(7): 1926-1933.

50. Ferdousi S, Mia AR. Serum levels of copper and zinc in newly diagnosed type-2 diabetic subjects. Mymensingh Med J. 2012;21(3):475-478.

51. Ekin S, Mert N, Gunduz H, Meral I. Serum sialic acid levels and selected mineral status in patients with type 2 diabetes mellitus. Biol Trace Elem Res. 2003;94(3):193-201.

52. Hasan BF. Status of some trace elements in Iraqi diabetic women and its relationship with lipid profile. IJSN. 2013;4(1):188-191

53. Siddiqui K, Bawazeer N, Scaria Joy S. Variation in macro and trace elements in progression of type 2 diabetes. Scientific World $J$. 2014;2014:Article ID 461591.

54. Adewumi MT, Njoku CH, Saidu Y, Abubakar MK, Shehu RA, Bilbis LS. Serum chromium, copper, and manganese levels of diabetic subjects in Katsina, Nigeria. Asian J Biochem. 2007;2:284-288.

55. Kazi TG, Afridi HI, Kazi N, Jamali MK, Arain MB, Jalbani N, Kandhro GA. Copper, chromium, manganese, iron, nickel, and zinc levels in biological samples of diabetes mellitus patients. Biol Trace Elem Res. 2008;122(1):1-8.

56. Forte G, Bocca B, Peruzzu A, et al. Blood metals concentration in type 1 and type 2 diabetics. Biol Trace Elem Res. 2013;156(1-3):79-90. 
57. Ekmekcioglu C, Prohaska C, Pomazal K, Steffan I, Schernthaner G, Marktl W. Concentrations of seven trace elements in different hematological matrices in patients with type 2 diabetes as compared to healthy controls. Biol Trace Elem Res. 2001;79(3):205-219.

58. Levy J, Gavin JR, Sowers JR. Diabetes mellitus: a disease of abnormal cellular calcium metabolism? Am J Med. 1994;96(3):260-273.
59. Levy J. Abnormal cell calcium homeostasis in type 2 diabetes mellitus. Endocrine. 1999;10(1):1-6.

60. Hermansen K, Mortensen LS, Hermansen ML. Combining insulins with oral antidiabetic agents: effect on hyperglycemic control, markers of cardiovascular risk and disease. Vasc Health Risk Manag. 2008;4(3):561-574.
Diabetes, Metabolic Syndrome and Obesity: Targets and Therapy is an international, peer-reviewed open-access journal committed to the rapid publication of the latest laboratory and clinical findings in the fields of diabetes, metabolic syndrome and obesity research. Original research, review, case reports, hypothesis formation, expert opinion and commentaries are all considered for publication. The manuscript management system is completely online and includes a very quick and fair peer-review system, which is all easy to use. Visit http://www.dovepress.com/testimonials.php to read real quotes from published authors.

Submit your manuscript here: https://www.dovepress.com/diabetes-metabolic-syndrome-and-obesity-targets-and-therapy-journal 